\title{
A Mixed Methods Exploration of Family Members'/Friends' Roles in a Self-Care Intervention for Depressive Symptoms
}

Tamara Sussman, ${ }^{1}$ Mark Yaffe, ${ }^{2}$ Jane Mccusker, ${ }^{3}$ Victoria Burns, ${ }^{1}$ Erin Strumpf, ${ }^{3,4}$ Maida Sewitch $^{5}$ and Eric Belzile ${ }^{6}$

\author{
${ }^{1}$ School of Social Work, McGill University, Montreal,Canada \\ ${ }^{2}$ Department of Family Medicine, McGill University,Montreal, Canada \\ ${ }^{3}$ Department of Epidemiology, Biostatistics and Occupational Health, McGill University, Montreal, Canada \\ ${ }^{4}$ Department of Economics, McGill University, Montreal,Canada \\ ${ }^{5}$ Department of Medicine, McGill University, Montreal,Canada \\ ${ }^{6}$ St. Mary's Hospital Research Center, Montreal, Canada
}

Corresponding author: Tamara Sussman, tel.: 514-398-2265, tamara.sussman@mcgill.ca; fax: (514) 398-4760

Funding: This work was supported by the Fonds de la Recherche du Québec - Santé (FRQS) [grant \#16384] May 2009-April 2013.

Objectives: The aims of this exploratory study were to examine the: 1) family/friend (F/F) support patients reported receiving and F/F reported providing to patients while participating in a self-care intervention (SCI) for depressive symptoms; and 2) associations between different types of F/F support and patients' use of the self-care tools in the SCI.

Methods: 57 patients aged $40+$ participating in an uncontrolled feasibility study of a SCI, completed structured telephone interviews about the support they received from F/F while participating in the SCI. $18 \mathrm{~F} / \mathrm{F}$ completed questionnaires on the support they provided to patients during the SCI. Seven F/F participated in a post study qualitative interview on their involvement in the SCI.

Results: $35 \%$ of patients reported receiving F/F support with the SCI. Patients use of the behavioral tools was positively associated with patients' report of $\mathrm{F} / \mathrm{F}$ support, and with F/F's report of instrumental support provided. F/F reported uncertainty about the type of support they should offer to patients in the SCI.

Discussion: $\mathrm{F} / \mathrm{F}$ involvement in SCIs for depressive symptoms may be helpful to patients and may foster adherence to these interventions. More research is warranted on the nature of such involvement from the perspective of patients and F/F.

\section{Keywords}

Self-care, depression, family support, chronic illnesses, adherence 


\section{INTRODUCTION}

Over the last 20 years there has been a trend to shift chronic disease management from physician as expert to physician-patient partnership where patients take an active role in managing their chronic symptoms with support from health practitioners and informal support networks. $\stackrel{1-4}{2}$ Referred to as "supported selfmanagement" or "supported self-care", this paradigm in chronic disease management shows promise for both clinical outcomes and cost-effectiveness. $\frac{\text { 5-7 }}{}$ Much empirical and conceptual consideration has been devoted to defining the roles, responsibilities and interactions between patients and physicians that motivate, educate and, empower patients to engage in self-care practices. ${ }^{2}, \underline{8-12}$ However, less attention has been given to involvement of family and friends $(\mathrm{F} / \mathrm{F}) . \underline{13}$

This is somewhat surprising given that social cognitive theory, the framework on which self-management is based, emphasizes that both personal factors (e.g. personal values, beliefs, self-efficacy) and environmental factors (e.g. formal health care providers, informal social network members $[\mathrm{F} / \mathrm{F}]$ ) have the potential to significantly influence health behavior. $\underline{14}$

Further, although it is increasingly recognized that feeling supported by $\mathrm{F} / \mathrm{F}$ is associated with better physical and mental health, the types of effective F/F involvement for particular health-related issues is less clear. This is especially the case for depressive symptoms where almost no research has been done. $\underline{3}, \underline{6}, \underline{15-17}$

We conducted a mixed-methods study among patients 40 years of age and over with depressive symptoms and comorbid chronic physical illnesses participating in a feasibility study of a self-care intervention (SCI) for depressive symptoms. F/F had no formally prescribed roles in the intervention. However patients had the option of sharing the self-care tools included in the intervention with F/F. We therefore explored 1) patients' perceptions of the support they received from F/F with self-care tools; 2) the relationship of perceived support to patients' use of self-care tools; 3 ) the relationship between $\mathrm{F} / \mathrm{F}$ reports of the emotional and instrumental support they provided and patients' use of self-care tools; and 4) how F/F perceived and experienced the SCI.

\section{BACKGROUND}

Managing a chronic illness often requires daily monitoring and symptom management, adhering to prescribed medications, coping with the impact of illness on daily life, and maintaining physical activity and specialized diet. $\frac{18}{}$ This is particularly challenging with depression as its very nature may compromise motivation. ${ }^{3} \underline{19}, \underline{20}$ Given the complexities involved in self-care, some patients rely on 
F/F to help them make decisions about seeking medical attention, adhere to medical recommendations, and emotionally cope with their conditions. $\underline{16}, \underline{18}, \underline{21-23}$

There is a small but growing literature on the roles F/F play in patients' experiences with and adherence to self- care with diabetes, heart failure, arthritis and asthma. ${ }^{17}, \underline{24}$ However, the literature is almost silent on F/F involvement with the management of depressive symptoms. In chronic physical conditions, illness-specific support such as adherence to diet for diabetes and, support in medical decisionmaking for heart failure seems more effective in promoting patients' self- care activities than general emotional support such as listening and providing empathy. Further, F/F support with activities of daily living (instrumental support) appears to positively impact both patients’ adherence to medical recommendations and experiences with care. Interestingly, F/F involvement can be experienced both positively as helpful and supportive, or negatively, as nagging and neglectful. $\underline{5-7, \underline{17}}, \underline{18}$, $\underline{24-27}$

While many patients appear to value and desire F/F involvement for many types of medical conditions (including depression) some have suggested that patients with depressive symptoms are more likely to report negative experiences with F/F involvement than patients managing physical conditions only. ${ }^{3}, \underline{27}$ This points to the importance of uncovering what types of $\mathrm{F} / \mathrm{F}$ involvement depressed patients value and benefit from. For example, F/F support with diet, medication adherence, and tasks of daily living, are common roles played by F/F for chronic physical conditions and have been shown to have some effect on patients' self-care practices and experiences. However, these forms of support have not been examined in the context of depression, making it difficult to determine the importance or necessity of these functions in self- management for depressive symptoms. Further, general emotional support, found to have little or no effect on health behaviors for patients managing chronic physical conditions, may play an important role in the management of depressive symptoms where some of the most difficult obstacles to self-care are emotional, such as feelings of hopelessness, lack of motivation, and shame. ${ }^{20}$

Building on the current literature, and addressing the gap on F/F support of patients managing depressive symptoms, this exploratory study sought to examine the role F/F play in a SCI offered to individuals with depressive symptoms and comorbid chronic physical illnesses, and to explore the relationship between different types of F/F involvement and use of self- care tools in the SCI. 


\section{METHODS}

We conducted an open, uncontrolled feasibility study of an SCI for depressive symptoms. All study procedures were approved by the St. Mary's Hospital Centre Research Ethics Committee.

\section{Sample and recruitment of patients}

In 2010-2011, 59 randomly selected family physicians in Montreal, Quebec agreed to have their patients aged 40 and over screened for depressive symptoms and a chronic physical illness. $\frac{28}{}$ Individuals having at least mild depressive symptoms (a score of five or above on the nine-item Patient Health Questionnaire - PHQ-9) $\stackrel{29}{ }$ and one or more of the study's targeted chronic physical illnesses (self-reported diagnosis of arthritis, hypertension, diabetes, heart disease, asthma, chronic obstructive pulmonary disease) were deemed eligible for participation at this stage. All eligible individuals were followed up by telephone by a research assistant who conducted a more extensive screening process. At this stage, patients were excluded from the study if they had suicidal plans, were receiving counseling or help from a mental health professional (once a month or more frequently), were unable to read either English or French, or had cognitive impairment (Blessed Orientation-MemoryConcentration test $(\mathrm{BOMC})$ score $<10) . \stackrel{30}{\underline{3}}$ Eligible patients were offered a six month SCI for depressive symptoms including a variety of self-care tools and the telephone support of a self-care coach.

\section{Sample and recruitment of family members/friends (F/F)}

At the time of enrolment, patients were invited to provide the name of one $\mathrm{F} / \mathrm{F}$ aged 18 and over who might be willing to participate in a survey on their observations and /or their involvement with the SCI. At 2 months, consenting F/F were asked to complete a brief mail-back questionnaire enquiring about their own socio-demographics, health status, and role in supporting patients with their depressive symptoms in general and with the SCI in particular. At the end of the six month follow-up, F/F who completed the baseline questionnaires were invited to participate in an end-of-study face-to-face qualitative interview.

\section{Self-care intervention}

Consenting patients received by mail a variety of self-care tools including 3 primarily informational tools (an educational pamphlet, a video/DVD, and information about an internet program) and 3 active behavioral tools based on cognitive-behavioral principles (a goal-setting workbook, an action plan, and a mood monitoring notebook). Patients also received a booklet on depression prepared specifically for $\mathrm{F} / \mathrm{F}$ that contained questions and answers about depression, the possible effects of depression on $\mathrm{F} / \mathrm{F}$, resources available to $\mathrm{F} / \mathrm{F}$, and the self-care material for depressive symptoms. Patients were not obligated to give the F/F this 
booklet. No other materials specific to F/Fs' involvement were included in the package. Each patient was assigned to one of two bilingual (English, French) selfcare coaches who were non-health care professionals, trained by our interdisciplinary research team to provide telephone-based support. Coaches provided weekly support for the first 3 months followed by monthly support for the remaining 3 months. The coaches did not provide therapy nor manage the patient's care. The latter was left to patients' physicians who continued to provide usual care. Coach support included, helping patients select self-care materials, responding to questions related to the material, and encouraging patients to continue with the tools (For a full description of the intervention offered, see. ${ }^{28}$ )

\section{MEASURES}

\section{Patient measures}

\section{Patient characteristics:}

Patient characteristics were assessed for descriptive purposes. Sociodemographic variables included: age, gender, living arrangement, marital status, level of education, place of birth, language spoken and household income. $\stackrel{31}{\text {, }} \underline{32}$ Health variables included: number of chronic conditions reported, and depressive symptoms as measured by the Patient Health Questionnaire (PHQ 9). The PHQ 9 asks patients to indicate how often in the past two weeks they have experienced each of nine symptoms on a four point scale. Options include not at all (0), several days (1), more than half the days (2) and nearly every day (3). Total scores ranging from 5-14 indicate mild-moderate depressive symptoms and scores 15 or greater indicate moderately severe -severe depressive symptoms. $\underline{\underline{33}}$

Patients' perceptions of F/F support with SCI:

Patients' perceptions of F/F support were assessed within a 4-category variable: (1) did not talk about depression or self-care tools with F/F; (2) talked about depression but not about the self-care tools with F/F;(3) talked about depression and the self-care tools with $\mathrm{F} / \mathrm{F}$ but did not receive support from $\mathrm{F} / \mathrm{F}$ with the tools; (4) talked about depression and the self-care tools with F/F and received F/F support with the tools. An additional open-ended question asked patients who indicated receipt of F/F support with the tools to describe the type of F/F support they found to be most helpful in supporting them in their use of tools. All comments were reviewed by TS and VB and coded as one of three categories developed from the responses:

(1) general emotional support which included comments made about emotional support offered (i.e. "He encouraged me and listened”; “Just encouraged me”; "Gave me a hug”), (2) tool specific support which included comments made about encouragement provided by F/F to use self-care tools (i.e. "She gave me ideas of how 
to go through the tools"; "He reminded me to watch the DVD"; "She encouraged me to follow the coach's instructions”) and (3) activity-specific support which included comments made about joint activities (i.e. "She invited me out to eat at a restaurant"; "She supported me to do exercise”; "We take walks together”).

Patient Report of self-care tool use:

Use of the self-care tools was assessed by asking patients to identify which tools they had tried and to what degree they had used the tools on a six-point Likert scale from 0 (not at all) to 5 (completed). Mean tool use scores were computed across the 3 informational and 3 behavioral tools, respectively, for 3 levels of use: $<1=$ minimal use, $1-2$ = moderate use; $3-5=$ high use.

\section{F/F measures}

\section{F/F characteristics:}

F/F characteristics were assessed for descriptive purposes. Relationship categories included: spouse/partner, adult child, sibling, or friend/other. Sociodemographic variables included: gender, age, place of birth, marital status, level of education, and household income. $\frac{31}{32}$ F/F health was assessed using the Health Survey Short Form-12 (SF-12) which includes both a physical component and a mental component. $\frac{34}{}$ Scores for each component can range from 0-100.

F/F perceptions of support provided:

F/F Emotional Involvement:

Emotional involvement of F/F was assessed with the Involvement Evaluation Questionnaire (IEQ) designed to capture both positive and negative forms of emotional involvement. $\frac{35}{5}$ The IEQ is a 31 - item questionnaire developed specifically for F/F supporting individuals with mental health issues and has shown good reliability and validity in a sample of family members supporting individuals with symptoms of depression..$^{20}$ The questionnaire includes four sub- scales tapping into the frequency in the last four weeks with which F/F (1) urged individuals to take care of themselves (eight items; possible scoring range 0-32); (2) experienced tension in their relationships with the individuals they are supporting (nine items; possible scoring range 0-36); (3) worried about the individuals they are supporting (six items; possible scoring range 0-24); (4) and supervised the individuals they are supporting to prevent self-harm (six items; possible scoring range 0-24). All items of the IEQ were scored on a 5-point Likert scale 0 (never), 1 (sometimes), 2(regularly), 3 (often) and 4 (always). Items scored between 0 (never) - 1 (sometimes) are considered to be reflective of low involvement. $\frac{20}{}$ Accordingly, in this paper, we defined low involvement for each sub-scale as an average item score of 1 or less. We adapted the questionnaire to exclude the concept of supervision of depressed individuals because 
the latter was considered specific to a population with more severe mental health issues than our study sample.

F/F Instrumental Support:

To further document F/F roles supporting patients, F/F were asked if they helped patients with any basic activities of daily living (ADLs), such as bathing, dressing and eating, and/or with instrumental activities of daily living (IADLs) such as shopping, cleaning and paying bills. Those who did not endorse any item were coded as not involved, while those who endorsed one or more were coded as involved.

\section{Qualitative Interview Guide}

A semi-structured interview guide was developed by TS and revised by the research team to ensure comprehensiveness and relevance to the project's goals. The guide aimed to elicit F/F experiences and involvement with patients' self-care practices in general and the SCI in particular. F/F recommendations to improve the SCI were probed. Three F/F were interviewed jointly by TS and a research assistant, and the remaining 4 by the research assistant only. Interviews were conducted in either English or French and lasted between 16 and 32 minutes.

\section{Data Analysis}

Descriptive statistics were used to characterize patients and F/Fs. To compare patients with and without F/F participation we used Chi-square or Fisher's exact test for categorical data $\stackrel{36}{ }$ and T-tests for continuous data. ${ }^{37}$ Given the small sample size in each group, we used the Kruskal Wallis test ${ }^{\frac{37}{3}}$ to compare the types of F/F involvement patients reported receiving with SCI and patients' reported self-care tool use; and the Mann-Whitney test $\frac{37}{}$ to examine potential associations between F/F reported instrumental support and emotional involvement and patient reported selfcare tool use. Quantitative data were analyzed using SAS version 9.2

All interviews were tape recorded, transcribed and analyzed using a threestaged method of analysis. In the first stage, each transcript was examined by VB who developed broad preliminary categories based on the general areas probed in the interview. Descriptive categories included recommendations made to improve $\mathrm{F} / \mathrm{F}$ involvement in SCI, F/F involvement in SCI and F/F involvement in self-care. In the second stage, TS and VB examined connections and patterns both within and between preliminary categories and within and between transcripts. At this stage of the analytic process uncertainty and confusion about F/F role emerged as a theme because it appeared to connect how F/F understood their involvement and F/F recommendations (connection between two preliminary categories). It was also discussed by multiple F/F (pattern between transcripts). Two additional themes 
providing general emotional support seen as important and F/F involvement in SCI requires more support and direction emerged during this second stage.

In the third and final stage the three themes and coded excerpts were presented and discussed with MY and JM who agreed that the themes were comprehensive and reliably represented comments made by participants.

\section{RESULTS}

254 patients were screened for participation in the study in family practices of which 98 (39\%) were eligible and interested in study participation. 38 (15\%) could not later be reached by telephone by the research team to assess for further eligibility, 51 (20\%) were no longer interested and 67 (26\%) were not eligible. 63 (64\%) of the 98 patients interested and eligible to participate, returned a signed consent form. The 35 (36\%) patients, who verbally agreed to participate but did not return a signed consent form (thereby excluding them from participation) did not differ significantly by age, sex, or PHQ-9 score from those who did. Among the 63 participants who signed a consent form at baseline and initiated participation in the study, 57 (90.5\%) completed a 2-month follow-up interview which asked them to report on their selfcare tool use and F/F involvement. Reasons for non-completion at 2 months were withdrawal or refusal (3) and unreachable within the time frame designated by the study protocol (3).

$24 \mathrm{~F} / \mathrm{F}$ were referred by patients, of whom18 (75\%) completed the baseline questionnaire, and 7 (29\%) participated in the end-of study qualitative interview. The qualitative sample size was reasonable given the specificity of the phenomena under investigation as it allowed us to achieve thematic saturation. $\frac{38}{}$ Reasons for refusal to participate in the qualitative interview included time commitments, loss of interest, and concerns that they had nothing further to offer because they saw themselves as only minimally supporting the patient.

Table 1 shows characteristics of the patient sample, and compares those with and without a participating $\mathrm{F} / \mathrm{F}$. The sample was predominantly female, educated at least to high school, born in Canada, and had mild-moderate depressive symptoms. Those with an F/F in the study were significantly more likely to be married. There were also substantive but non-significant differences in the percentages of patients living alone and with 3 or more chronic diseases.

Table 2 shows characteristics of F/F sample and their perspectives on the support provided to patients. The sample of F/F comprised a mix of different types of relatives and friends. Their SF-12 scores were consistent with average levels of physical and mental health. $\frac{39}{9}$ About two-thirds provided instrumental support to the 
patients and few provided basic ADL support. Average emotional involvement scores for urging, worrying and tension were suggestive of low involvement. ${ }^{20}$ Patient perspectives: Support provided by F/F and self-caret tool use

Table 3 shows patients' perspectives on the support provided by F/F and the relationship between support provided and patient tool use. 81\% (46/57) and 74\% (42/57) of patients respectively reported speaking to their F/F about their depressed mood and the self-care tools. 35\% (20/57) affirmed some form of support from their $\mathrm{F} / \mathrm{F}$ with the SCI. Amongst the 20 patients receiving support from their F/F with the SCI, 55\% (11) identified general emotional support, 30\% (6) tool-specific support, and 15\% (3) activity-specific support as the most helpful form of support received. $\mathrm{F} / \mathrm{F}$ involvement and support in use of the tools was significantly associated with greater patient use of the behavioral, but not informational tools.

\section{F/F perspectives: Types of $F / F$ involvement and patient self- care tool use}

Table 4 shows the associations between F/F-reported instrumental support and emotional involvement with patient-reported use of the self-care tools. Only instrumental support was significantly associated with greater use of the behavioral tools. Patients' report of greater use of the behavioral tools was associated with higher scores on two components of F/F reported emotional involvement, urging (providing encouragement) and tension (arguing with patient to participate in selfcare) but this trend did not achieve significance levels.

\section{Results of Qualitative Interviews with F/F}

Demographics of F/F who participated in qualitative interviews

Two friends, one godson, two daughters and two spouses participated in F/F qualitative interviews, ranging in ages from 40-70+. All were Canadian born. Most were women and had completed more than high school.

Providing general emotional support seen as important to F/F

All F/F described their main role as one of providing general emotional support. Initiating contact and actively listening to patients were seen as important means through which F/F communicated their care and concern for patients. As one daughter states: "Oh yes she loves to be called - she wants that, she wants me to do the calling. She feels - well no, she'll call me if she doesn't hear from me but she would prefer if I show the initiative” Similarly, a female spouse says, "Yes, that's right; I am the 'psychiatrist'. Well, not exactly but still, I listen. Sometimes I give him ideas, but it's really in his hands, because after all, it's up to him to interpret things" [translation].

Uncertainty and confusion about F/F role in SCI

Although F/F were comfortable providing general emotional support to patients, many wondered how and if they should play a more active role in SCI. 
Apprehensions included changing the nature of their relationship, lack of qualifications, and concern that their involvement might hinder rather than help the person with their depression.

A daughter expressed worry that playing a helping role could interfere with her father-daughter relationship. She states, "You don't want it to be too I guess, like oh I'm you know I don't want to be analyzing everything father does every day, every second of the day. I don't want to be looking at my father as a mouse, you know what I mean?”

A female friend expressed confusion and concern about her competence to provide appropriate and helpful support. She states: “It's hard. It's hard because you know, I see her suffering, and I can't do anything. I'm not a professional, I can't, I can talk to her so much but you know I don't ask the right questions or say the right things all the time - sometimes I say the wrong thing, you know.”

F/F involvement in SCI requires more support and direction

While most F/F had not played an active role in SCI, many thought that they might have been more comfortable becoming involved had they been provided with direction and support. Recommendations to improve involvement included sending information about self-care tools directly to F/F and having a self-care coach provide one telephone call to F/F to inform them about the study and to educate them what their role might look like in the intervention. Most F/F felt that this awareness and direction would have allowed them to invite opportunities for their involvement. The godson noted 'I think it might've made a difference because I could've spoken to her everyday about it, you know, 'how are you feeling today'...'is it better than yesterday or worse than yesterday?' So yeah, I think if I had received the material also it might've made a big difference, I could've motivated her more”. Similarly, a daughter thought it would have been helpful to know more about the intervention because she believes her mother needs someone to motivate her: "Yeah if you give her stuff, the material is going to go somewhere nicely, neatly in the corner and she's never going to look at it. She needs someone to tell her 'you have to do this' the way I do on the phone”.

\section{DISCUSSION}

\section{Summary of Main Findings}

In this mixed-methods study, we explored the role F/F play in a SCI offered to individuals with depressive symptoms and comorbid chronic physical illnesses, and the relationship between different types of $\mathrm{F} / \mathrm{F}$ involvement and use of the self- care tools. Overall, 35\% of patients reported receiving some form of F/F support with the 
SCI. The most helpful forms of support patients reported receiving were general emotional support, followed by tool-specific support, and activity-specific support.

Patients` use of the behavioral tools was associated with patient reported receipt of some form of F/F support with tools, and with F/F reported provision of instrumental support. Qualitative interviews with F/F revealed that F/F were positive about the general emotional support they provided but were uncertain about the extent and type of involvement they should offer to patients with SCI. Some F/F felt that more direct information about the self-care tools and their potential role may have improved their confidence to inquire about self-care and motivate patients with tool use.

\section{Interpretation and Prior Literature}

The majority of patients reported discussing their depression and the self-care tools with F/F. Previous research indicates adults with depression are hesitant to discuss their condition with others for fear of being pressured to take medication or being labeled as “mentally ill”, $\underline{19}, \underline{40}$. Patients who engage in a SCI for depressive symptoms may be a biased sample that is inherently more open to F/F involvement than adults with depressive symptoms in general.

The patients who reported that they received F/F support with their tool use were more likely to continue using behavioral tools than those who did not. The most helpful supports identified by patients who reported receiving support were: general emotional support (listening, giving a hug) followed by tool -specific (providing ideas of how to go through tools) and activity specific support (inviting them to dinner, supporting them in doing their exercises). The literature on F/F involvement in self-management for physical conditions has identified an important link between illness specific support (e.g. diet planning for diabetes) and patient adherence to selfcare regimes. $\frac{17}{}$ Our findings suggest that general emotional support and direct involvement in self-care activities such as exercising, going out for dinner, and providing ideas for using self-care materials may represent illness specific support for depressive symptoms.

F/F reported provision of instrumental support such as shopping and housekeeping was also associated with patients' use of behavioral tools. This form of assistance has been found to support adherence to self-care in other studies, particularly when instrumental support is linked to self- care behaviors such as assisting with the purchase of groceries to support a particular dietary regime. $\frac{17}{}$ Our small sample of F/F did not allow for analyses of the association between different types of instrumental supports and patients' self-care practices. Future research should examine different types of instrumental support typically offered to patients with depressive symptoms and their differential relationship with self-care practices. 
Such data could be helpful in providing F/F with direction on roles and responsibilities that may help adherence to self-care for depressive symptoms. It would also be helpful for patient outcomes as use of behavioral tools has been associated with improvement in depressive symptoms. $\underline{28}$

Although patient reported F/F support with SCI was associated with patient reported behavioral tool use, and many patients reported talking to F/F about the selfcare materials, most patients did not report receiving F/F support with self-care tools. Our qualitative data provided some insight into this trend highlighting that F/F were confused about the role they should play with the SCI, worrying that their support, while well intentioned, may cause more harm than good. According to the literature patients with depressive symptoms desire F/F support but are also more likely to experience this support negatively than patients with physical conditions only, ${ }^{27}$ F/F involvement with SCI may therefore need to be less ad hoc than that which occurred in our project. Specifically, there may be value to structured dialogue between patients and $\mathrm{F} / \mathrm{F}$ at the outset about roles and expectations, and such discussion could be re-visited periodically during the SCI. This might help to decrease risk of patient resentment and $\mathrm{F} / \mathrm{F}$ uncertainty.

\section{Limitations}

The major study limitation is limited generalizability of our findings given the modest numbers of participating patients and F/Fs. A second study limitation is misclassification given that the study measures on F/F support may not have been sufficiently sensitive to capture F/F involvement and support. For example, patients were asked to report if they received support for their tool use but were not asked if they perceived this support to be helpful, harmful or neutral. Patients were also not asked to identify the type of support they received making it difficult to identify how patients understood the receipt of F/F support with tool use. Further, the two forms of support reported as most helpful by patients (general emotional support and activity specific support) were not explored in the questionnaire administered to F/F.

\section{Implications for Clinical Practice and Future Research}

This study examined the ways F/F become involved in SCI for patients with depressive symptoms. However, our intervention did not actively encourage F/F involvement. Instead we relied on patients to engage F/F as they saw fit. Our findings indicated that many patients elected to discuss the SCI with F/F; however F/F lacked the confidence or direction to respond. While it may be preliminary to recommend that patients experiences with and adherence to SCI for depression would improve with more structured F/F involvement, the idea of a more formalized approach for depression care, as suggested above, merits study. 
Findings from the literature on chronic physical conditions do suggest that providing $\mathrm{F} / \mathrm{F}$ and patients with opportunities to discuss care expectations and training $\mathrm{F} / \mathrm{F}$ in approaches that support patient autonomy are superior to providing F/F with education only. These interventions are also acceptable to both patients and F/F and appear to support patients' adherence to medical regimes. $\stackrel{18,22, \text { 27, }}{\text { 41-44 }}$ Future research exploring the acceptability of these approaches for depression SCI and their impact on patients' and F/F's experiences of care and outcomes is suggested.

Our study is the first to report on the different types of F/F involvement and support in a SCI aimed at helping patients manage their depressive symptoms. Further exploration of F/F roles and interventions that may help both F/F and persons with depressive symptoms participate in and benefit from SCI is warranted. 


\section{REFERENCES}

1. Bilsker D, Goldner EM, Anderson E. Supported self-management: A simple, effective way to improve depression care. Can J Psychiatry 2012;57(4):203-209.

2. Bodenheimer T, Lorig K, Holman H, et al. Patient self-management of chronic disease in primary care. JAMA 2002;288:2469-2475.

3. McCusker J, Latimer E, Cole M, et al. The nature of informal caregiving for medically ill older people with and without depression. Int J Geriatr Psychiatry 2009;24(3):239-246.

4. Matire LM, Stephens MAP, Druley JA, et al. Negative reactions to received spousal care: Predictors and consequences of miscarried support. Health Psychol 2002;21(2):167-176.

5. Dunbar S, Clark P, Quinn C, et al. Family influences on heart failure selfcare and outcomes. J Cardiovasc Nurs 2008;23(3):258-265.

6. Luttik ML, Jaarsma T, Moser D, et al. The importance and impact of social support on outcomes in patients with heart failure: An overview of the literature. J Cardiovasc Nurs 2005;20(3):162-169.

7. Coyne JC, Rohrbaugh MJ, Shoham V, et al. Prognostic importance of marital quality for survival of congestive heart failure. Am J Cardiol 2001;88(5):526-529.

8. Martin CM. Chronic disease and illness care: Adding principles of family medicine to address ongoing health system redesign. Can Fam Physician 2007;53(12):2086-2091.

9. Katon W, Von Korff M, Lin E, et al. Rethinking practitioner roles in chronic illness: The specialist, primary care physician, and the practice nurse. Gen Hosp Psychiatry 2001;23(3):138-144.

10. Ryan RM, Patrick H, Deci EL, et al. Facilitating health behaviour change and its maintenance: Interventions based on self-determination theory. The European Health Psychologist 2008;10(1):2-5.

11. Deci EL, Ryan RM. Self-determination theory in health care and its relations to motivational interviewing: A few comments. Int J Behav Nutr Phys Act 2012;9(1):24.

12. Ryan RM, Patrick H, Deci EL, et al. Facilitating health behaviour change and its maintenance: Interventions based on self-determination theory. Health Psychol Rev 2008;10(1):2-5.

13. Piette JD. Moving beyond the notion of 'self' care. Chronic Illn 2010;6(1):3-6. 
14. Bandura A. Social foundations of thought and action: A social cognitive approach. Englewood Cliffs, NJ: Prentice Hall, 1986.

15. Hillier J, Rooksby E. Habitus: A sense of place. 2nd ed. Burlington, VT: Ashgate Publishing Company, 2005.

16. Sayers SL, Riegel B, Pawlowski S, et al. Social support and self-care of patients with heart failure. Ann Behav Med 2008;35(1):70-79.

17. DiMatteo MR. Social support and patient adherence to medical treatment: A meta-analysis. Health Psychol 2004;23(2):207-218.

18. Sebern MD, Woda A. Shared care dyadic intervention: Outcome patterns for heart failure care partners. West J Nurs Res 2012;34(3):289-316.

19. Smith M. Stigma. Adv Psychiatr Treat 2002;8(5):317-323.

20. van Wijngaarden B, Schene AH, Koeter MWJ. Family caregiving in depression: Impact on caregivers' daily life, distress, and help seeking. $J$ Affect Disord 2004;81(3):211-222.

21. Gleeson-Kreig J, Bernal H, Woolley S. The role of social support in the selfmanagement of diabetes mellitus among a hispanic population. Public Health Nurs 2002;19(3):215-222.

22. Rosland A-M, Piette JD. Emerging models for mobilizing family support for chronic disease management: A structured review. Chronic Illn 2010;6(1):721.

23. Toljamo M, Hentinen M. Adherence to self-care and social support. J Clin Nurs 2001;10(5):618-627.

24. Gallant MP. The influence of social support on chronic illness selfmanagement: A review and directions for research. Health Educ Behav 2003;30(2):170-195.

25. Riegel B, Lee CS, Dickson VV, et al. An update on the self-care of heart failure index. J Cardiovasc Nurs 2009;24(6):485.

26. Sebern M, Riegel B. Contributions of supportive relationships to heart failure self-care. Eur J Cardiovasc Nurs 2009;8(2):97-104.

27. Rosland AM, Heisler M, Choi HJ, et al. Family influences on selfmanagement among functionally independent adults with diabetes or heart failure: Do family members hinder as much as they help? Chronic Illn 2010;6(1):22-33.

28. McCusker J, Cole MG, Yaffe M, et al. A feasibility study of a telephonesupported self-care intervention for depression among adults with a comorbid chronic physical illness in primary care. Ment Health Fam Med In press:000-000. 
29. Löwe B, Unützer J, Callahan CM, et al. Monitoring depression treatment outcomes with the patient health questionnaire-9. Med Care 2004;42(12):1194-1201.

30. Katzman R, Brown T, Fuld P, et al. Validation of a short orientationmemory-concentration test of cognitive impairment. Am J Psychiatry 1983;140(6):734-739.

31. Statistics Canada. Health Statistics Division, Data Liberation Initiative (Canada), McGill University. Electronic Data Resources Service., et al. Canadian community health survey- cycle 1.2, mental health and well-being (2002). Ottawa, Ontario, Québec: Statistics Canada, Health Statistics Division distributed through Sherlock, Cooperative Data Access System for Quebec University Libraries; 2003. Available from:

http://sherlock.crepuq.qc.ca/cgibin/sherlock.pl?langue=E;action=LAE;region=Canada;region=Autres.

32. Statistics Canada. Health Statistics Division, Data Liberation Initiative (Canada), McGill University. Electronic Data Resources Service, et al. Canadian community health survey- cycle 2.1- 2003. Ottawa, Ontario, Québec: Statistics Canada, Health Statistics Division distributed through Sherlock, Cooperative Data Access System for Quebec University Libraries; 2004. Available from: http://sherlock.crepuq.qc.ca/cgibin/sherlock.pl?langue=E;action=LAE;region=Canada;region=Autres.

33. Kroenke K, Spitzer RL, Williams JBW. The phq-9: Validity of a brief depression severity mjournal of general internal medicineeasure. J Gen Intern Med 2001;16(9):606-613.

34. Ware Jr JE, Kosinski M, Keller SD. A 12-item short-form health survey: Construction of scales and preliminary tests of reliability and validity. Med Care 1996;34(3):220-233.

35. van WijnGaarden B, Schene A, Koeter M, et al. Caregiving in schizophrenia: Development, internal consiconsistency and reliability of the involvement evaluation questionnaire - european version. $\mathrm{Br} J$ Psychiatry 2000;177(39):s21-s27.

36. Fleiss JL, Levin B, Paik MC. Statistical methods for rates and proportions, third edition. Shewart WA, Wilks SS, editors. Hoboken, NJ: John Wiley \& Sons, Inc., 2003.

37. Sheskin DJ. Handbook of parametric and nonparametric statistical procedures. Boca Raton, FL: Chapman and Hall/CRC, 2004.

38. Patton M. Qualitative research and evaluation methods. Thousant Oaks, California: Sage, 2002. 
39. Burdine JN, Felix MRJ, Abel AL, et al. The sf-12 as a population health measure: An exploratory examination of potential for application. Health Serv Res 2000;35(4):885-904.

40. Berkman LF. The role of social relations in health promotion. Psychosomatics 1995;57(3):245-254.

41. Fisher L. Research on the family and chronic disease among adults: Major trends and directions. Fam Syst Health 2006;24(4):373.

42. Fava GA, Ruini C, Rafanelli C, et al. Six-year outcome of cognitive behavior therapy for prevention of recurrent depression. Am J Psychiatry 2004;161(10):1872-1876.

43. Britt E, Hudson SM, Blampied NM. Motivational interviewing in health settings: A review. Patient Educ Couns 2004;53:147-155.

44. Clark AM, Freydberg CN, McAlister FA, et al. Patient and informal caregivers' knowledge of heart failure: Necessary but insufficient for effective self-care. European Journal of Heart Failure 2009;11(6):617-621. 
Sussman et al.

Page 18 of 21

Table 1 : Characteristics of Patients with and without a Family Member/ Friend in the Study (n=57)

\begin{tabular}{lcccc}
\hline Characteristic & $\begin{array}{c}\text { Overall } \\
(\mathrm{n}=57)\end{array}$ & $\begin{array}{c}\text { Without F/F } \\
(\mathrm{n}=39)\end{array}$ & $\begin{array}{c}\text { With F/F } \\
(\mathrm{n}=18)\end{array}$ & $\begin{array}{c}\text { p-value } \\
\text { (chi-squar })\end{array}$ \\
\hline Sociodemographic: & & & & \\
Age (mean(sd)) & $60.6(10.5)$ & $60.6(10.3)$ & $60.6(11.2)$ & $0.992^{*}$ \\
Female (\%) & 73.7 & 69.2 & 83.3 & $0.342^{* *}$ \\
Lives alone (\%) & 28.1 & 35.9 & 11.1 & 0.053 \\
Married/common-law (\%) & 45.6 & 35.9 & 66.7 & 0.030 \\
High school or greater (\%) & 84.2 & 84.6 & 83.3 & 1.000 \\
Born in Canada(\%) & 75.4 & 71.8 & 83.3 & 0.511 \\
French-speaking (\%) & 50.9 & 48.7 & 55.6 & 0.631 \\
Low income (\%) & 26.7 & 29.0 & 21.4 & 0.725
\end{tabular}

\section{Health measures:}

Number of chronic diseases:

$$
2 \text { chronic diseases(\%) }
$$

33.3

35.9

27.8

$3+$ chronic diseases $(\%)$

21.1

25.6

11.1

Depressive Symptoms:

Mild-Moderate $\quad<15(\%)$

Moderately Severe-Severe $\geq 15$ (\%)

70.2

76.9

55.6

\section{$*$ T-test}

**Fisher's exact test 
Sussman et al.

Variables

\begin{tabular}{|c|c|c|}
\hline & $\underline{\mathbf{n}}$ & $(\%)$ \\
\hline \multicolumn{3}{|l|}{ Relationship with FM } \\
\hline Spouse/partner & 7 & (38.9) \\
\hline Adult child & 3 & $(16.7)$ \\
\hline Brother/sister & 2 & $(11.1)$ \\
\hline Friend/other & 6 & (33.3) \\
\hline $\begin{array}{l}\text { Female } \\
\text { (missing) }\end{array}$ & $\begin{array}{l}12 \\
(1)\end{array}$ & $(70.6)$ \\
\hline \multicolumn{3}{|l|}{ Age } \\
\hline $30-49$ & 5 & $(29.4)$ \\
\hline $50-59$ & 7 & $(41.2)$ \\
\hline $60-79$ & 5 & (29.4) \\
\hline (missing) & (1) & \\
\hline Born in Canada & 14 & $(82.4)$ \\
\hline (missing) & (1) & \\
\hline \multicolumn{3}{|l|}{ Marital status } \\
\hline Single, widowed or divorced & 7 & $(40.2)$ \\
\hline Married or living common-law & 10 & (59.8) \\
\hline (missing) & (1) & \\
\hline \multicolumn{3}{|l|}{ Education } \\
\hline High school or less & 5 & $(27.8)$ \\
\hline More than high school & 13 & $(72.2)$ \\
\hline \multicolumn{3}{|l|}{ Household Income } \\
\hline Less than $\$ 25,000$ & 5 & (27.8) \\
\hline$\$ 25,000$ to less than $\$ 75,000$ & 8 & (44.4) \\
\hline$\$ 75,000$ and more & 4 & $(22.2)$ \\
\hline Unknown & 1 & $(5.6)$ \\
\hline \multicolumn{3}{|l|}{ ADL Support } \\
\hline Instrumental support & 12 & $(66.7)$ \\
\hline Basic support & 2 & $(11.1)$ \\
\hline SF-12 & $\underline{\mathbf{n}}$ & mean(sd) \\
\hline Mental mean(SD) & $\overline{16}$ & $\overline{50.6(8.0)}$ \\
\hline Physical mean(SD) & 16 & $53.0(7.9)$ \\
\hline \multicolumn{3}{|l|}{ Involvement score } \\
\hline Urging mean(SD) & 17 & $1.1(0.7)$ \\
\hline Tension mean(SD) & 18 & $0.5(0.4)$ \\
\hline Worrying mean(SD) & 17 & $0.9(0.6)$ \\
\hline
\end{tabular}

\section{Table 2: Family Member/Friend Demographics (n=18)}

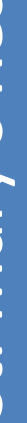


Table 3: Patient Perspectives: Partial Support Provided by F/F and Patients' Self-Care Tool Use (n=57)

Family members involvement and support with tools

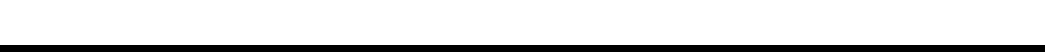

\section{Use of informational tools**}

n Median

p value

[Q1-Q3]

0.107

$\begin{array}{lll}11 & 0.67 & {[0.33 ; 1.67]} \\ 14 & 1.67 & {[1.67 ; 2.67]} \\ 12 & 2.00 & {[0.83 ; 3.33]} \\ 20 & 1.67 & {[1.17 ; 3.17]}\end{array}$

Use of behavioral tools** Median

[Q1-Q3]

p value*

$<0.001$

Did not talk about depressed mood

Talked about depressed mood but not about the tools

Talk about the tools but no support

Talk about the tools and have received support

$\begin{array}{ll}0.67 & {[0.00 ; 1.00]} \\ 1.00 & {[0.67 ; 2.67]} \\ 1.50 & {[0.67 ; 1.83]} \\ 3.00 & {[2.00 ; 3.50]}\end{array}$

* Kruskal-Wallis test

$* *<1$ minimal, 1-2 moderate, 3-5: high 
Table 4: Type of F/F Involvement and Patients' Self-Care Tool Use ( $n=18)$

\begin{tabular}{|c|c|c|c|c|c|c|c|c|}
\hline \multirow{2}{*}{$\begin{array}{c}\text { Family/Friend } \\
\text { Baseline Involvement }\end{array}$} & & \multirow[b]{2}{*}{$\mathbf{n}$} & \multicolumn{3}{|c|}{ Use of informational tools** } & \multicolumn{3}{|c|}{ Use of behavioral tools** } \\
\hline & & & Median & [Q1-Q3] & p value* & Median & [Q1-Q3] & p value* \\
\hline Instrumental support & & & & & 0.362 & & & 0.012 \\
\hline & no & 6 & 1.7 & {$[1.3 ; 2.0]$} & & 0.5 & {$[0.0 ; 0.7]$} & \\
\hline & yes & 12 & 1.5 & {$[0.0 ; 1.7]$} & & 2.0 & {$[0.8 ; 3.2]$} & \\
\hline
\end{tabular}

Emotional involvement:

\begin{tabular}{|c|c|c|c|c|c|c|}
\hline Urging & & & & & & \\
\hline Low Involvement & $\leq 8$ & 10 & 1.7 & {$[1.3 ; 1.7]$} & 1 & {$[0.7 ; 2.3]$} \\
\hline High Involvement & $>8$ & 7 & 1 & {$[0.0 ; 1.7]$} & 1.7 & {$[0.0 ; 3.3]$} \\
\hline
\end{tabular}

Tension***

na

na

\begin{tabular}{|c|c|c|c|c|c|c|c|}
\hline Low Involvement & $\leq 9$ & 17 & 1.7 & {$[0.3 ; 1.7]$} & & 1 & {$[0.7 ; 2.7]$} \\
\hline High Involvement & $>9$ & 1 & 1.7 & [na] & na & 2.3 & \\
\hline
\end{tabular}

Worrying

0.329

Low Involvement $\quad \leq 6 \quad 12 \quad 1.3 \quad[0.2 ; 1.7]$

$0.7 \quad[0.2 ; 3.2]$

High Involvement $>6 \quad 5$

$1.7 \quad[1.7 ; 1.8]$

$1.7 \quad[1.7 ; 2.3]$

* Mann-Whitney test

** (same as Table 3)

Emotional involvement was dichotomized as low (scores less than or equal to the sum of items in a sub-scale) and high (scores greater than the sum of items in a sub-scale)

*** Analysis not done due to inadequate sample size in high involvement group 